\title{
Truncation Error Estimate on Random Signals by Local Average
}

\author{
Gaiyun $\mathrm{He}^{1}$, Zhanjie Song ${ }^{2, \star}$, Deyun Yang ${ }^{3}$, and Jianhua Zhu ${ }^{4}$ \\ ${ }^{1}$ School of Mechanical Engineering, Tianjin University, \\ Tianjin 300072, China \\ hegaiyun@tju.edu.cn \\ ${ }^{2}$ School of Science, Tianjin University, Tianjin 300072, China \\ zhanjiesong@tju.edu.cn \\ ${ }^{3}$ Department of Information Science, Taishan College, \\ Taian 271000, China \\ nkuydy@163. com \\ ${ }^{4}$ National Ocean Technique Center, Tianjin 300111, China \\ besmile@263.net
}

\begin{abstract}
Since signals are often of random characters, random signals play an important role in signal processing. We show that the bandlimited wide sense stationary stochastic process can be approximated by Shannon sampling theorem on local averages. Explicit truncation error bounds are given.
\end{abstract}

Keywords: stochastic process, random Signals, local averages, truncation error, Shannon sampling theorem.

\section{Introduction and the Main Result}

The Shannon sampling theorem plays an important role in signal analysis as it provides a foundation for digital signal processing. It says that any bandlimited function $f$, having its frequencies bounded by $\pi \mathcal{W}$, can be recovered from its sampled values taken at instances $k / \mathcal{W}$, i.e.

$$
f(t)=\sum_{k=-\infty}^{+\infty} f\left(\frac{k}{\mathcal{W}}\right) \operatorname{sinc}(\mathcal{W} t-k),
$$

where $\operatorname{sinc}(t)=\sin \pi t /(\pi t), t \neq 0$, and $\operatorname{sinc}(0)=1$.

This equation requires values of a signal $f$ that are measured on a discrete set. However, due to its physical limitation, say the inertia, a measuring apparatus may not be able to obtain exact values of $f$ at epoch $t_{k}$ for $k=0,1,2, \cdots$. Instead, what a measuring apparatus often gives us is a local averages of $f$ near $t_{k}$ for each $k$. The sampled values defined as local averages may be formulated by the following equation

\footnotetext{
* Corresponding author. Supported by the Natural Science Foundation of China under
} Grant (60572113, 40606039) and the Liuhui Center for Applied Mathematics. 


$$
\left\langle f, u_{k}\right\rangle=\int f(x) u_{k}(x) d x
$$

for some collection of averaging functions $u_{k}(x), k \in \mathbb{Z}$, which satisfy the following properties,

$$
\operatorname{supp} u_{k} \subset\left[x_{k}-\frac{\sigma}{2}, x_{k}+\frac{\sigma}{2}\right], \quad u_{k}(x) \geq 0, \quad \text { and } \quad \int u_{k}(x) d x=1 .
$$

Where $u_{k}$ for each $k \in \mathbb{Z}$ is a weight function characterizing the inertia of measuring apparatus. Particularly, in an ideal case, the function is given by Dirac $\delta$-function, $u_{k}=\delta\left(\cdot-t_{k}\right)$, because $\left\langle f, u_{k}\right\rangle=f\left(t_{k}\right)$ is the exact value of $t_{k}$.

The local averaging method in sampling was studied by a number of papers [1]- 6] form 1994 to 2006.

The associated truncation error of (10) is defined dy

$$
R_{N}^{f}=f(t)-\sum_{k=-N}^{+N} f\left(\frac{k}{\mathcal{W}}\right) \operatorname{sinc}(\mathcal{W} t-k)=\frac{\sin \pi \mathcal{W} t}{\pi} \sum_{|k|>N}(-1)^{k} \frac{f(k / \mathcal{W})}{\mathcal{W} t-k}
$$

But on the one hand, we can not finish a infinite number of terms in practise, we only approximated signal functions by a finite number of terms. Which is called truncation error deal with bounds by a number of papers [7. [15].

On the other hand, since signals are often of random characters, random signals play an important role in signal processing, especially in the study of sampling theorems. For example, a signal of speech, where the random portion of the function may be white noise or some other distortion in the transmission channel, perhaps given via a probability distribution. So there are a lots of papers on this topic too. Such as [16]-[24]. Now we give truncation error bounds random signals by local averages.

Before stating the results, let us introduce some notations. $L^{p}(\mathbb{R})$ is the space of all measurable functions on $\mathbb{R}$ for which $\|f\|_{p}<+\infty$, where

$$
\begin{aligned}
\|f\|_{p} & :=\left(\int_{-\infty}^{+\infty}|f(u)|^{p} d u\right)^{1 / p}, \quad 1 \leq p<\infty, \\
\|f\|_{\infty} & :=\operatorname{ess}_{u \in \mathbb{R}}|f(u)|, \quad p=\infty .
\end{aligned}
$$

$B_{\pi \mathcal{W}, p}$ is the set of all entire functions $f$ of exponential type with type at most $\pi \mathcal{W}$ that belong to $L^{2}(\mathbb{R})$ when restricted to the real line 25 . By the PaleyWiener Theorem, a square integrable function $f$ is band-limited to $[-\pi \mathcal{W}, \pi \mathcal{W}]$ if and only if $f \in B_{\pi \mathcal{W}, 2}$.

Given a probability space $(\Omega, \mathrm{A}, \mathrm{P})[26$, a real-valued stochastic process $X(t):=X(t, \omega)$ defined on $\mathbb{R} \times \Omega$ is said to be stationary in weak sense if $E\left[X(t)^{2}\right]<\infty, \forall t \in \mathbb{R}$, and the autocorrelation function

$$
R_{X}(t, t+\tau):=\int_{\Omega} X(t, \omega) X(t+\tau, \omega) d P(\omega)
$$

is independent of $t \in \mathbb{R}$, i.e., $R_{X}(t, t+\tau)=R_{X}(\tau)$. 
A weak sense stationary process $X(t)$ is said to be bandlimited to an interval $[-\pi \mathcal{W}, \pi \mathcal{W}]$ if $R_{X}$ belongs to $B_{\pi \mathcal{W}, p}$ for some $1 \leq p \leq \infty$.

Now we assume that $u_{k}$ which are given by (3) satisfy the following properties.

i) $\operatorname{supp} u_{k} \subset\left[\frac{k}{\mathrm{~W}}-\sigma_{k}^{\prime}, \frac{k}{\mathrm{~W}}+\sigma_{k}^{\prime \prime}\right]$, where $\sigma / 4 \leq \sigma_{k}^{\prime}, \sigma_{k}^{\prime \prime} \leq \sigma / 2, \sigma$ are positive constants;

ii) $\quad u_{k}(t) \geq 0, \int u_{k}(t) d t=1$;

iii) $m=\inf _{k \in Z}\left\{m_{k}\right\}$, where $m_{k}:=\int_{k / \mathrm{W}-\sigma / 4}^{k / \mathrm{W}+\sigma / 4} u_{k}(t) d t$.

In this cases, The associated truncation error of random signals $X(t, \omega)$ is defined dy

$$
R_{N}^{X}=X(t)-\sum_{k=-N}^{+N}\left\langle X, u_{k}\right\rangle \operatorname{sinc}(\mathrm{W} t-k) .
$$

where the autocorrelation function of the weak sense stationary stochastic process $X(t, \omega)$ belongs to $B_{\pi \mathcal{W}, 2}$, and $\mathrm{W}>\mathcal{W}>0$.

The following results is proved by Belyaev and Splettstösser in 1959 and 1981, respectively.

Proposition A. [16, Theorem 5]) If the autocorrelation function of the weak sense stationary stochastic process $X(t, \omega)$ belongs to $B_{\pi \mathcal{W}, 2}$, for $\mathrm{W}>\mathcal{W}>0$, we have

$$
\begin{aligned}
E\left[\left|R_{N}^{X *}\right|^{2}\right] & =E\left(\left|X(t, \omega)-\sum_{k=-N}^{N} X\left(\frac{k}{\mathrm{~W}}, \omega\right) \operatorname{sinc}(\mathrm{W} t-k)\right|^{2}\right) \\
& \leq \frac{16 R_{X}(0)(2+|t| \mathrm{W})^{2}}{\pi^{2}(1-\mathcal{W} / \mathrm{W})^{2} N^{2}}
\end{aligned}
$$

Proposition B. [17, Theorem 2.2]) If the autocorrelation function of the weak sense stationary stochastic process $X(t, \omega)$ belongs to $B_{\pi \mathrm{W}, p}$ for some $1 \leq p \leq 2$ and $\Omega>0$, then

$$
\lim _{N \rightarrow \infty} E\left(\left|X(t, \omega)-\sum_{k=-N}^{N} X\left(\frac{k}{\mathrm{~W}}, \omega\right) \operatorname{sinc}(\mathrm{W} t-k)\right|^{2}\right)=0 .
$$

For this case, we have the following result.

Theorem C. If the autocorrelation function $R_{X}$ of a weak sense stationary stochastic process $X(t, \omega)$ belongs to $B_{\mathcal{W}, 2}$, for $\mathrm{W}>\mathcal{W}>0$ and $2 / \delta \geq N \geq 100$, we have

$$
E\left[\left|R_{N}^{X}\right|^{2}\right] \leq\left(14.80\left\|R^{\prime \prime}\right\|_{\infty}+\frac{32 R_{X}(0)(2+|t| \mathrm{W})^{2}}{\pi^{2}(1-\mathcal{W} / \mathrm{W})^{2}}\right)\left(\frac{\ln N}{N}\right)^{2},
$$

where $\left\{u_{k}(t)\right\}$ is a sequence of continuous functions defined by (5). 
Or, in other words

$$
E\left[\left|R_{N}^{X}\right|^{2}\right]=O\left(\frac{\ln N}{N}\right)^{2}, \quad N \rightarrow \infty
$$

\section{Proof of the Main Result}

Let us introduce some preliminary results first.

Lemma D. 27] One has for $q^{\prime}>1,1 / p^{\prime}+1 / q^{\prime}=1$, and $\mathrm{W}>0$,

$$
\sum_{k=-\infty}^{\infty}|\operatorname{sinc}(\mathrm{W} t-k)|^{q^{\prime}} \leq 1+\left(\frac{2}{\pi}\right)^{q^{\prime}} \frac{q^{\prime}}{q^{\prime}-1}<p^{\prime} .
$$

Lemma E. 26] If a stationary stochastic process $X(t, \omega), t \in[a, b]$ is continuous in mean square, $f(t), g(t)$ are continuous function on $[a, b]$, then

$$
E\left[\int_{a}^{b} f(s) X(s) d s \cdot \int_{a}^{b} g(t) X(t) d t\right]=\int_{a}^{b} \int_{a}^{b} f(s) g(t) R(s-t) d s d t .
$$

Lemma F. Suppose that the autocorrelation function $R_{X}$ of the weak sense stationary stochastic process $X(t, \omega)$ belongs to $B_{\pi \mathrm{W}, 2}$ and $\mathrm{W}>0$, and satisfies $R_{X}^{\prime \prime}(t) \in C(\mathbb{R})$. Let

$$
\begin{aligned}
& D\left(\frac{j}{\mathrm{~W}} ; \frac{\delta}{2}\right) \\
:= & \sup _{\substack{\left|\delta^{*}\right| \leq \frac{\delta}{2} \\
\left|\delta^{* *}\right| \leq \frac{\delta}{2}}}\left|R_{X}\left(\frac{j}{\mathrm{~W}}\right)-R_{X}\left(\frac{j}{\mathrm{~W}}-\delta^{* *}\right)-R_{X}\left(\frac{j}{\mathrm{~W}}+\delta^{*}\right)+R_{X}\left(\frac{j}{\mathrm{~W}}+\delta^{*}-\delta^{* *}\right)\right| \\
= & \sup _{\substack{\left|\delta^{*}\right| \leq \frac{\delta}{2} \\
\left|\delta^{* *}\right| \leq \frac{\delta}{2}}}\left|\int_{-\delta^{* *}}^{0} \int_{\delta^{*}}^{0} R_{X}^{\prime \prime}\left(\frac{j}{\mathrm{~W}}+u+v\right) d u d v\right| .
\end{aligned}
$$

Then we have for $r, N \geq 1$,

$$
\sum_{j=-2 N}^{+2 N}\left[D\left(\frac{j \pi}{\Omega} ; \frac{\delta}{2}\right)\right]^{r} \leq(4 N+1)\left(\left\|R_{X}^{\prime \prime}(t)\right\|_{\infty}\right)^{r}\left(\frac{\delta}{2}\right)^{2 r} .
$$

Proof. Since $R_{X}$ is even and $R_{X}^{\prime \prime}(t) \in C(\mathbb{R})$, we have

$$
\begin{aligned}
\sum_{j=-2 N}^{+2 N}\left[D\left(\frac{j \pi}{\Omega} ; \frac{\delta}{2}\right)\right]^{r} & =\left[D\left(0 ; \frac{\delta}{2}\right)\right]^{r}+2 \sum_{j=1}^{2 N}\left[D\left(\frac{j \pi}{\Omega} ; \frac{\delta}{2}\right)\right]^{r} \\
& \leq(4 N+1)\left(\left\|R_{X}^{\prime \prime}(t)\right\|_{\infty}\right)^{r}\left(\frac{\delta}{2}\right)^{2 r} .
\end{aligned}
$$

which completes the proof. 
Proof of Theorem C. From Proposition A, Proposition B and Lemma E we have

$$
\begin{aligned}
& E\left[\left|R_{N}^{X}\right|^{2}\right]=E\left[\left|X(t, \omega)-\sum_{k=-N}^{N} \int_{k / \mathrm{W}-\delta_{k}^{\prime}}^{k / \mathrm{W}+\delta_{k}^{\prime \prime}} u_{k}(t) X(t, \omega) \operatorname{dtsinc}(\mathrm{W} t-k)\right|^{2}\right] \\
& =E\left[\mid X(t, \omega)-\sum_{k=-N}^{N} X\left(\frac{k}{\mathrm{~W}}, \omega\right) \operatorname{sinc}(\mathrm{W} t-k)\right. \\
& +\sum_{k=-N}^{N} X\left(\frac{k}{\mathrm{~W}}, \omega\right) \operatorname{sinc}(\mathrm{W} t-k) \\
& \left.-\left.\sum_{k=-N}^{N} \int_{k / \mathrm{W}-\delta_{k}^{\prime}}^{k / \mathrm{W}+\delta_{k}^{\prime \prime}} u_{k}(t) X(t, \omega) \operatorname{dsinc}(\mathrm{W} t-k)\right|^{2}\right] \\
& =2 E\left[\left|R_{N}^{X *}\right|^{2}\right] \\
& +2 E\left[\left|\sum_{k=-N}^{N}\left[X\left(\frac{k}{\mathrm{~W}}, \omega\right)-\int_{k / \mathrm{W}-\delta_{k}^{\prime}}^{k \pi / \Omega+\delta_{k}^{\prime \prime}} u_{k}(t) X(t, \omega) d t\right] \operatorname{sinc}(\mathrm{W} t-k)\right|^{2}\right] \\
& =2 E\left[\left|R_{N}^{X *}\right|^{2}\right]+2 E\left[\mid \sum_{k=-N}^{N}\left[\int_{-\delta_{k}^{\prime}}^{\delta_{k}^{\prime \prime}} X\left(\frac{k}{\mathrm{~W}}, \omega\right) u_{k}\left(\frac{k}{\mathrm{~W}}+s\right) d s\right.\right. \\
& \left.\left.-\int_{-\delta_{k}^{\prime}}^{\delta_{k}^{\prime \prime}} u_{k}(k / \mathrm{W}+t) X(k \pi / \Omega+t, \omega) d t\right]\left.\operatorname{sinc}(\mathrm{W} t-k)\right|^{2}\right] \\
& =2 E\left[\left|R_{N}^{X *}\right|^{2}\right]+2 \sum_{k=-N}^{N} \sum_{j=-N}^{N} \\
& \left(\int_{-\delta_{k}^{\prime}}^{\delta_{k}^{\prime \prime}} \int_{-\delta_{k}^{\prime}}^{\delta_{k}^{\prime \prime}} E\left[X\left(\frac{k}{\mathrm{~W}}, \omega\right) X\left(\frac{j}{\mathrm{~W}}, \omega\right)\right] u_{k}\left(\frac{k}{\mathrm{~W}}+u\right) u_{j}\left(\frac{j}{\mathrm{~W}}+v\right) d u d v\right. \\
& -\int_{-\delta_{k}^{\prime}}^{\delta_{k}^{\prime \prime}} \int_{-\delta_{k}^{\prime}}^{\delta_{k}^{\prime \prime}} E\left[X\left(\frac{k}{\mathrm{~W}}, \omega\right) X\left(\frac{j}{\mathrm{~W}}+v, \omega\right)\right] u_{k}\left(\frac{k}{\mathrm{~W}}+u\right) u_{j}\left(\frac{j}{\mathrm{~W}}+v\right) d u d v \\
& -\int_{-\delta_{k}^{\prime}}^{\delta_{k}^{\prime \prime}} \int_{-\delta_{k}^{\prime}}^{\delta_{k}^{\prime \prime}} E\left[X\left(\frac{k}{\mathrm{~W}}+u, \omega\right) X\left(\frac{j}{\mathrm{~W}}, \omega\right)\right] u_{k}\left(\frac{k}{\mathrm{~W}}+u\right) u_{j}\left(\frac{j}{\mathrm{~W}}+v\right) d u d v \\
& \left.+\int_{-\delta_{k}^{\prime}}^{\delta_{k}^{\prime \prime}} \int_{-\delta_{k}^{\prime}}^{\delta_{k}^{\prime \prime}} E\left[X\left(\frac{k}{\mathrm{~W}}+u, \omega\right) X\left(\frac{j}{\mathrm{~W}}+v, \omega\right)\right] u_{k}\left(\frac{k}{\mathrm{~W}}+u\right) u_{j}\left(\frac{j}{\mathrm{~W}}+v\right) d u d v\right) \\
& \cdot|\sin c(\mathrm{~W} t-k)||\sin c(\mathrm{~W} t-j)| \\
& =2 E\left[\left|R_{N}^{X *}\right|^{2}\right]+2 \sum_{k=-N}^{N} \sum_{j=-N}^{N} \int_{-\delta_{k}^{\prime}}^{\delta_{k}^{\prime \prime}} \int_{-\delta_{k}^{\prime}}^{\delta_{k}^{\prime \prime}}\left[R_{X}\left(\frac{(k-j)}{\mathrm{W}}\right)-R_{X}\left(\frac{(k-j)}{\mathrm{W}}-v\right)\right. \\
& \left.-R_{X}\left(\frac{(k-j)}{\mathrm{W}}+u\right)+R_{X}\left(\frac{(k-j)}{\mathrm{W}}+u-v\right)\right] u_{k}\left(\frac{k}{\mathrm{~W}}+u\right) u_{j}\left(\frac{j}{\mathrm{~W}}+v\right) d u d v
\end{aligned}
$$


$\cdot|\operatorname{sinc}(\mathrm{W} t-k)||\sin c(\mathrm{~W} t-j)|$

$$
\begin{aligned}
\leq & 2 E\left[\left|R_{N}^{X *}\right|^{2}\right]+2 \sum_{k=-N}^{N} \sum_{j=-N}^{N} \int_{-\delta_{k}^{\prime}}^{\delta_{k}^{\prime \prime}} \int_{-\delta_{k}^{\prime}}^{\delta_{k}^{\prime \prime}} D\left(\frac{(k-j)}{\mathrm{W}} ; \frac{\delta}{2}\right) \\
& u_{k}\left(\frac{k}{\mathrm{~W}}+u\right) u_{j}\left(\frac{j}{\mathrm{~W}}+v\right) d u d v \cdot|\sin c(\mathrm{~W} t-k)||\operatorname{sinc}(\mathrm{W} t-j)| \\
= & 2 E\left[\left|R_{N}^{X *}\right|^{2}\right]+2 \sum_{k=-N}^{N} \sum_{j=-N}^{N} D\left(\frac{(k-j)}{\mathrm{W}} ; \frac{\delta}{2}\right)|\operatorname{sinc}(\mathrm{W} t-k)||\operatorname{sinc}(\mathrm{W} t-j)|
\end{aligned}
$$

Using Hölder's inequality and Lemma D, we have

$$
\begin{aligned}
& \sum_{k=-N}^{N} \sum_{j=-N}^{N} D\left(\frac{(k-j)}{\mathrm{W}} ; \frac{\delta}{2}\right)|\operatorname{sinc}(\mathrm{W} t-k)| \cdot|\operatorname{sinc}(\mathrm{W} t-j)| \\
\leq & \left(\sum_{k=-N}^{N}\left|\sum_{j=-N}^{N} D\left(\frac{(k-j)}{\mathrm{W}} ; \frac{\delta}{2}\right)\right| \operatorname{sinc}(\mathrm{W} t-j)||^{p^{*}}\right)^{1 / p^{*}} \\
& \cdot\left(\sum_{k=-N}^{N}|\operatorname{sinc}(\mathrm{W} t-k)|^{q^{*}}\right)^{1 / q^{*}} \\
\leq & \left(p^{*}\right)^{1 / q^{*}}\left(\sum_{k=-N}^{N}\left|\sum_{j=-N}^{N} D\left(\frac{(k-j)}{\mathrm{W}} ; \frac{\delta}{2}\right)\right| \operatorname{sinc}(\mathrm{W} t-j)||^{p^{*}}\right)^{1 / p^{*}} \\
\leq & p^{*}\left(\sum_{k=-N}^{N}\left|\sum_{j=-N}^{N} D\left(\frac{(k-j)}{\mathrm{W}} ; \frac{\delta}{2}\right)\right| \operatorname{sinc}(\mathrm{W} t-j)||^{p^{*}}\right)^{1 / p^{*}},
\end{aligned}
$$

where $1 / p^{*}+1 / q^{*}=1$. By Hausdorff-Young inequality [28, page176] and Lemma $\mathrm{F}$, we have

$$
\begin{aligned}
& \left(\sum_{k=-N}^{N}\left|\sum_{j=-N}^{N} D\left(\frac{(k-j)}{\mathrm{W}} ; \frac{\delta}{2}\right)\right| \operatorname{sinc}(\mathrm{W} t-j)||^{p^{*}}\right)^{1 / p^{*}} \\
\leq & \left(\sum_{j=-2 N}^{2 N}\left[D\left(\frac{j}{\mathrm{~W}} ; \frac{\delta}{2}\right)\right]^{r^{*}}\right)^{1 / r^{*}}\left(\sum_{j=-2 N}^{2 N}|\operatorname{sinc}(\mathrm{W} t-j)|^{s^{*}}\right)^{1 / s^{*}} \\
\leq & (4 N+1)^{1 / r^{*}}\left\|R_{X}^{\prime \prime}(t)\right\|_{\infty}\left(\frac{\delta}{2}\right)^{2}\left(\sum_{j=-2 N}^{2 N}|\operatorname{sinc}(\Omega t-j \pi)|^{s^{*}}\right)^{1 / s^{*}}
\end{aligned}
$$




$$
\leq(4 N+1)^{1 / r^{*}}\left\|R_{X}^{\prime \prime}(t)\right\|_{\infty}\left(\frac{1}{N}\right)^{2}\left(\sum_{j=-\infty}^{\infty}|\operatorname{sinc}(\Omega t-j \pi)|^{s^{*}}\right)^{1 / s^{*}},
$$

where $0 \leq 1 / s^{*}+1 / r^{*}-1=1 / p^{*}$. Let $r^{*}=\ln N / 2$. Notice that $N \geq 100$, we have

$$
(4 N+1)^{1 / r^{*}}<\left(\frac{401 N}{400}\right)^{2 / \ln N} \leq 7.40
$$

Let $s^{*}=2 r^{*} /\left(2 r^{*}-1\right)$ and $s^{\prime}=2 r^{*}$. Then $1 / s^{*}+1 / s^{\prime}=1$ and $p^{*}=2 r^{*}=\ln N$. By Lemma E, we have

$$
\left(\sum_{j=-\infty}^{\infty}|\operatorname{sinc}(\Omega t-j \pi)|^{s^{*}}\right)^{1 / s^{*}} \leq s^{\prime}=p^{*}=\ln N
$$

Hence

$$
E\left[\left|R_{N}^{X}\right|^{2}\right] \leq\left(14.80\left\|R^{\prime \prime}\right\|_{\infty}+\frac{32 R_{X}(0)(2+|t| \mathrm{W})^{2}}{\pi^{2}(1-\mathcal{W} / \mathrm{W})^{2}}\right)\left(\frac{\ln N}{N}\right)^{2} .
$$

This completes the proof.

\section{References}

1. Gröchenig, K., Reconstruction algorithms in irregular sampling, Math. Comput., 59(1992), 181-194.

2. Butzer, P. L., Lei, J., Errors in truncated sampling series with measured sampled values for non-necessarily bandlimited functions, Funct. Approx. Comment. Math. 26(1998), 18-32,

3. Butzer, P. L., Lei, J., Approximation of signals using measured sampled values and error analysis, Commun.Appl.Anal., 4(2000), 245-255.

4. Sun, W., Zhou, X., Reconstruction of bandlimited functions from local averages, Constr. Approx., 18(2002), 205-222.

5. Sun, W., Zhou, X., Reconstruction of bandlimited signals from local averages, IEEE Trans. Inform. Theory, 48(2002), 2955-2963.

6. Song, Z., Yang, S., Zhou, X., Approximation of signals from local averages, Applied Mathematics Letters, 19(2006), 1414-1420.

7. Yao, K., Thomas, J. B., On truncation error bounds for sampling representations of band-limited signals, IEEE Trans. Aerosp. Electron. syst., vol.AEs-2(1966), 640647.

8. Jagerman, D., Bounds for truncation error of the sampling expansion, SIAM J. Appl. Math., vol.14(1966), 714-723.

9. Brown, J. L., Bounds for truncation error in sampling expansion of band-limited signals, IEEE Trans. Inform. Theory, vol.IT-15(1969), 440-444. 
10. Piper, H. S. Jr., Bounds for truncation error in sampling expansion of finite energy band-limited signals, IEEE Trans. Inform. Theory, vol.IT-21(1975), 482-485.

11. Piper, H. S. Jr., Best asymptotic bounds for truncation error in sampling expansion of band-limited functions, IEEE Trans. Inform. Theory, vol.IT-21(1975), 687-690.

12. Butzer, P. L., Engels, W., Scheben, U., Magnitude of the truncation error in sampling expansion of band-limited signals, IEEE Trans. Acoustics, Speech, and Signal Processing, vol.ASSP-30(6)(1982), 906-912.

13. Butzer, P. L., Engels, W., On the implementation of the Shannon sampling series for band-limited signals, IEEE Trans. Inform. Theory, vol.IT-29(2)(1983), 314-318.

14. Bucci, O. M., Massa, G. D., The truncation error in the application of sampling series to electromagnetic problems, IEEE Trans. Antennas and Propagation, vol.36(7)(1988), 941-949.

15. Machiraju, R., Yagel, R. K., Reconstruction error characterization and control: a sampling Theory approach, IEEE Trans. Visual. Comput. Graphics, vol.2(4)(1996), 364-378.

16. Belyaev, Y. K., Analytic random processes, Theory Probab. Appl. IV(1959) 437-444.

17. Splettstösser, W., sampling series approximation of continuous weak sense stationary processes, Information and Control 50(1981), 228-241.

18. Balakrishnan, A. V., A note on the sampling principle for continuous signals, IRE Trans. Inform. Theory IT-3(1957), 143-146.

19. Lloyd, S. P., A sampling theorem for stationary (wide sense) stochastic processes, Trans. Amer. Math. Soc. 92(1959), 1-12.

20. Stens, R. L. , Error estimates for sampling sums based on convolution integrals, Information and Control 45(1980), 37-47.

21. Butzer, P.L., Splettstösser, W. and Stens R. L., The sampling theorem and linear prediction in signal analysis, Jber. d. Dt. Math.-Verein., 90(1988), 1-70.

22. Olenko, A. YA., Pogany, T. K., A precise bound for the error of interpolation of stochastic processes, Theor. Probability and Math Statist. vol.71(2005), 151-163.

23. Song, Z., Zhou, X., He, G., Error estimate on no-bandlimited radom signals by local averages, LNCS3991(2006), 822-825.

24. Song, Z., Sun, W., Yang, S., Zhu, G., Approximation of Weak Sense Stationary Stochastic Processes from Local Averages, Science in China: Series A Math. $50(4)(2007), 457-463$.

25. Zayed, A.I., Butzer, P.L., Lagrange interpolation and sampling theorems, in "Nonuniform Sampling, Theory and Practice", Marvasti,F., Ed., Kluwer Academic, 2001, 123-168.

26. Li, Z., Wu, R., A course of studies on stochastic processes, High Education Press, 1987(in chinese).

27. Splettstösser, W., Stens, R. L., Wilmes, G., on the approximation of the interpolating series of G. Valiron, Funct. Approx. Comment. Math. 11(1981), 39-56.

28. Pinsky, M. A., Introduction to Fourier analysis and wavelets, Wadsworth Group. Brooks/Cole.(2002) Comput., 59, 181-194. 